\title{
Morphological and Physiological Features of a Set of Spinal Substantia Gelatinosa Neurons Defined by Green Fluorescent Protein Expression
}

\author{
Adam W. Hantman, ${ }^{1}$ Anthony N. van den Pol, ${ }^{2}$ and Edward R. Perl ${ }^{1}$ \\ ${ }^{1}$ Department of Cell and Molecular Physiology, University of North Carolina at Chapel Hill, Chapel Hill, North Carolina 27599, and ${ }^{2}$ Department of \\ Neurosurgery, Yale University School of Medicine, New Haven, Connecticut 06520
}

\begin{abstract}
The spinal substantia gelatinosa (SG) is known to be involved in the manipulation of nociceptive and thermal primary afferent input; however, the interrelationships of its neuronal components are poorly understood. As a step toward expanding understanding, we took a relatively unique approach by concentrating on a set of SG neurons selectively labeled by green fluorescent protein (GFP) in a transgenic mouse. These GFP-expressing SG neurons prove to have homogenous morphological and electrophysiological properties, are systematically spaced in the SG, contain GABA, receive C-fiber primary afferent input, and upregulate c-Fos protein in response to noxious stimuli. Together, the properties established for these GFP-labeled neurons are consistent with a modular SG organization in which afferent activity related to nociception or other C-fiber signaling are subject to integration/modulation by repeating, similar circuits of neurons.
\end{abstract}

Key words: C-fiber; dorsal horn; GABA; nociception; spinal; substantia gelatinosa; pain

\section{Introduction}

Establishing the functional architecture of the spinal substantia gelatinosa (SG) (lamina II) is challenging. Developmental mapping has shown that the neuronal composition and synaptic arrangements of the SG originate from multiple lineages of neuronal progenitor cells (Lee and Jessell, 1999; Müller et al., 2002). Morphological and electrophysiological studies indicate that the developed SG is composed of numerous distinctive types of neurons that have different responses to sensory input (Ramon y Cajal, 1909; Gobel, 1975; 1978; Kumazawa and Perl, 1978; Price et al., 1979; Todd and Lewis, 1986; Grudt and Perl, 2002). The many putative synaptic mediators associated with SG neurons suggest synaptic complexity and functional distinctions (Todd and Spike, 1993; Ren and Ruda, 1994).

Despite limitations in our knowledge of SG architecture, there is compelling evidence that the SG is involved in mechanisms related to pain, itch, and temperature senses. Thinly myelinated $(\mathrm{A} \delta)$ and unmyelinated $(\mathrm{C})$ primary afferent fibers concentrate their central projections in the SG (Light and Perl, 1979a,b; Sugiura et al., 1986). These fine afferent fibers are known to be involved in signaling noxious, temperature, itch, and certain tactile

Received Sept. 15, 2003; revised Nov. 7, 2003; accepted Nov. 7, 2003.

This work was supported by Research Grant NS-10321 from the National Institute of Neurological Disorders and Stroke of the National Institutes of Health. We thank Ms. Bonnie Taylor-Blake for excellent assistance with immunocytochemical studies and Mr. Kirk McNaughton for superior histological work. We are grateful to Mrs. Sherry Joseph for valuable help in preparation of this manuscript. Peter Somogyi graciously provided a GABA antibody for use in these experiments.

Correspondence should be addressed to Dr. Edward R. Perl, Department of Cell and Molecular Physiology, University of North Carolina at Chapel Hill, 5109 Neuroscience Research Building, Campus Box 7545, Chapel Hill, NC 27599. E-mail: erp@med.unc.edu.

D01:10.1523/JNEUROSCI.4221-03.2004

Copyright $\odot 2004$ Society for Neuroscience $\quad 0270-6474 / 04 / 240836-07 \$ 15.00 / 0$ stimuli (Perl, 1984; Tuckett and Wei, 1987a,b; Vallbo et al., 1999). Furthermore, suprasegmental centers capable of modulating nociception and pain (Proudfit and Anderson, 1975) send projections to the SG (Dahlström and Fuxe, 1965; Clark and Proudfit, 1991).

Progress in knowledge of the relationship between SG intrinsic neurons and somatosensory mechanisms would be advanced by systematic characterizations of particular SG neuronal categories and their relationships to dorsal root input. The absence of obvious anatomical patterning in the arrangement of SG neuronal subtypes makes it difficult to collect electrophysiological and morphological observations on a given category of neuron. Immunohistochemical studies frequently do not provide adequate information on either the classes of neurons expressing particular antigens or the cellular function of the antigens. To circumvent these difficulties, we targeted a specific population of SG neurons for study. The transgenic mouse [prion promotor (Prp)-GFP] used for this set of experiments expresses green fluorescent protein (GFP) in a limited set of SG cells under the control of the prion promoter (van den Pol et al., 2002). Although the basis of this selective expression pattern has not yet been determined, we interpret it to result from a combination of promoter and integration site effects. We report here characterization of the following: (1) the distribution of the SG-GFP neurons in the spinal cord, (2) the synaptically active agent they express, (3) their morphology, (4) their electrophysiological features, and (5) their response to dorsal root afferent input. Evidence is provided that suggests the SG-GFP neurons are activated by noxious stimulation. A preliminary report of this study was presented at the Society for Neuroscience Annual Meeting in 2002 (Hantman et al., 2002). 

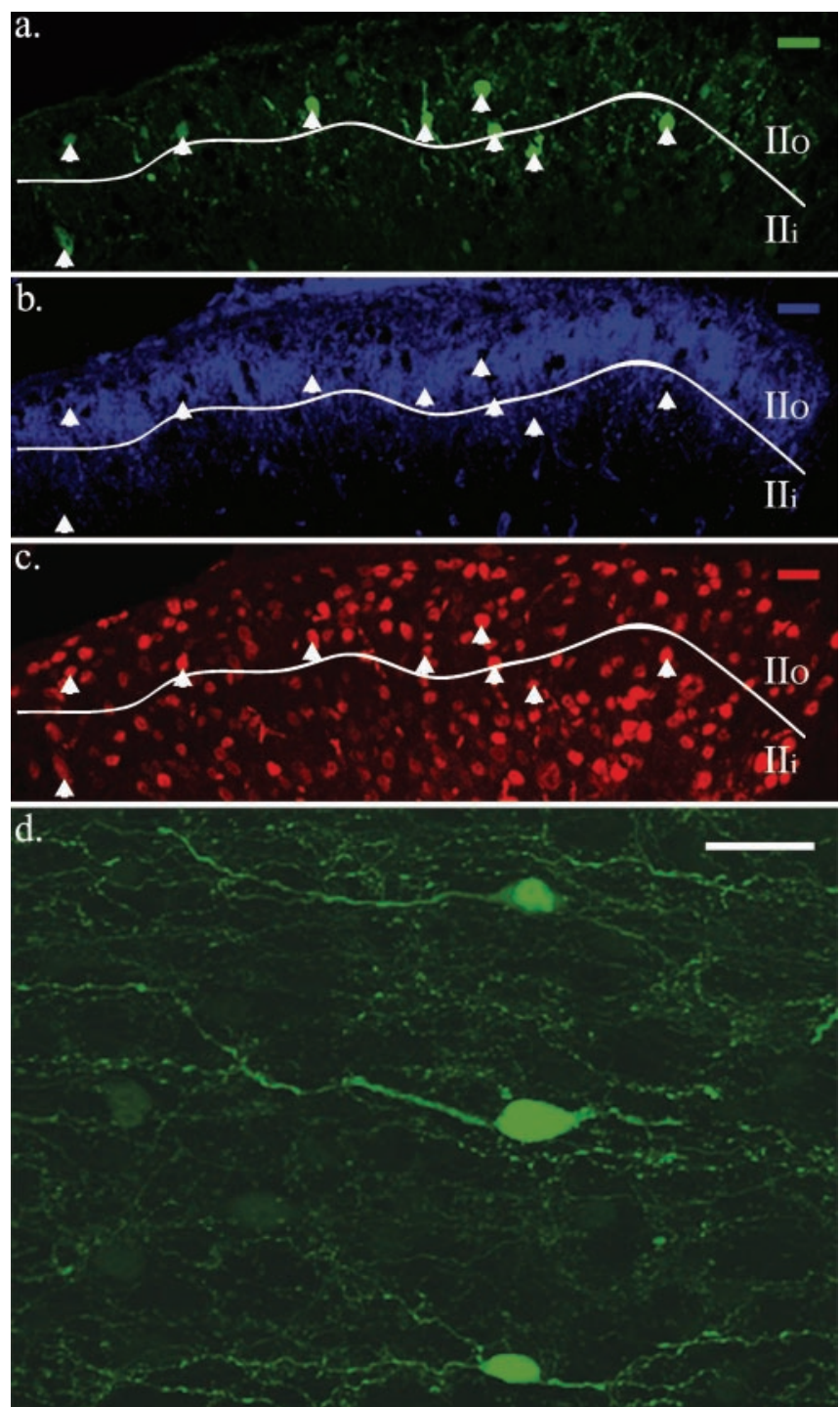

Figure 1. GFP-expressing cells in the spinal cord are predominately located in the SG. $a$ SG-GFP cells (green) are concentrated in lamina $\|_{0}$ and are evenly distributed in the mediallateral axis of the spinal cord. $b$, The majority of $S G-G F P$ neurons are in $S G$ regions delineated by IB4 binding (blue signal). C, SG-GFP cells are neurons. Red represents immunoreactivity to a NeuN antibody. Boundary indicated between lamina $\|_{i}$ and $\|_{0}$ (line) is approximate. Arrows demark SG-GFP somata. Dorsal is shown at top. $d$, Higher magnification view of SG-GFP cells in a horizontal section showing their similar morphologies. Medial is shown at the top, and rostral is shown to the left. Scale bars, $25 \mu \mathrm{m}$.

\section{Materials and Methods}

Animals

All of the procedures were approved by the Institutional Animal Care and Use Committee of the University of North Carolina at Chapel Hill and Yale University. The adult White Swiss-Webster transgenic mice used in this study were designed to express enhanced, red-shifted, mammalian codon-corrected GFP under control of the mouse prion promoter (van den Pol et al., 2002). Prp-GFP animals were crossed, and the strain proved stable for $>10$ generations in terms of consistent GFP expression in dorsal horn neurons. The electrophysiological attributes, including membrane potential, input resistance, response to transmitters, spike threshold, and synaptic activity of neurons expressing GFP expression under control of the prion promoter, was comparable with observations on non-GFP neurons, suggesting that, under these conditions, the expression of GFP has little effect on physiological properties (van den Pol et al., 2002).
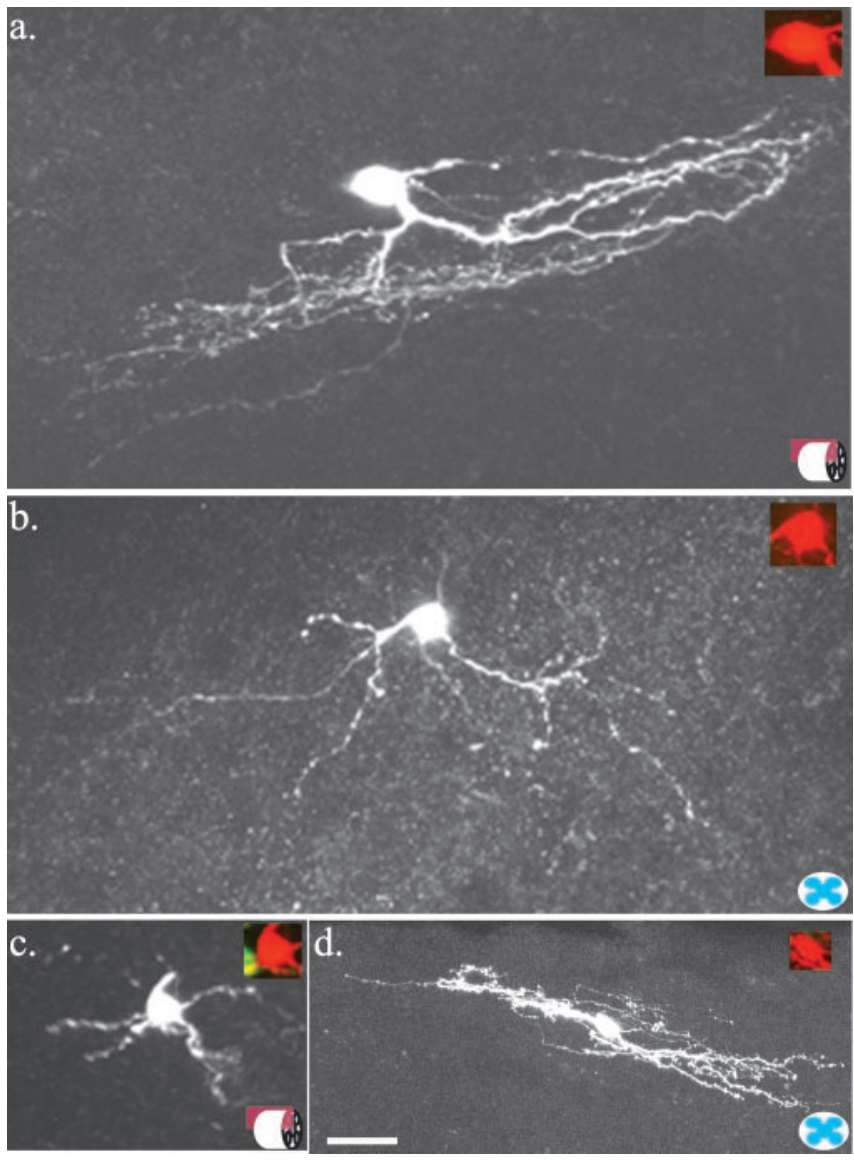

Figure 2. Examples of the morphology of neurons recorded from the center of the SG that did not express GFP. Maximum projection confocal images of biocytin-labeled neurons. $a$, Vertical cell in a sagittal spinal section (represented by icon at bottom right). $b$, Medial-lateral cell in a transverse spinal section. c, Radial cell in a transverse spinal section (represented by icon at bottom right). $d$, Central cell in a sagittal spinal section. Insets are merged images of GFP signal ( green) and biocytin signal (red); absence of overlap indicates that the cell did not contain GFP. Dorsal is shown at the top. Scale bar, $25 \mu \mathrm{m}$.

\section{Immunocytochemistry}

Spinal tissue was fixed either by immersion (slices) or perfusion with $4 \%$ paraformaldehyde. For use of the Somogyi and Sigma (St. Louis, MO) GABA antibodies, the perfusion fixative was $2.5 \%$ glutaraldehyde and $1 \%$ paraformaldehyde. After a minimum of $8 \mathrm{hr}$ of immersion fixation or $2 \mathrm{hr}$ of postfixation for perfused tissue, the tissue was cryoprotected using a 30\% sucrose phosphate buffer solution. Cryostat-cut spinal cord sections were incubated with primary antibodies selective for substance $\mathrm{P}$ (1:1000; DiaSorin, Stillwater, MN), neuronal nuclei (NeuN) (1:100; Chemicon, Temecula, CA), neuron-specific enolase (1:100; Chemicon), GABA (1:250; Chemicon), GABA (1:3000; Somogyi; gift from P. Somogyi, University of Oxford, Medical Research Council-Anatomical Neuropharmacology Unit, Oxford, UK), GABA (1:6000; Sigma), calcitonin gene-related peptide (1:1000; Peninsula, San Carlos, CA), and c-Fos (1: 200; Genosys, The Woodlands, TX). Fluorophore-conjugated secondary antibodies raised against the appropriate species were then incubated with the tissue. Isolectin B4 (IB4) binding was detected by sequential incubation of tissue sections with biotinylated Griffonia simplicifolia-IB4 (IB4; Vector Laboratories, Burlingame, CA) and streptavidin-Cy5 (Zymed, San Francisco, CA). For GABA preabsorption controls, equal volumes of GABA-BSA conjugate $(2.5 \mathrm{mg} / \mathrm{ml})$ and the GABA antibody were combined before the primary incubation step and applied to the tissue.

Biocytin immunocytochemistry. After a minimum of $20 \mathrm{~min}$ in the whole-cell, tight-seal patch-clamp configuration, the biocytin-filled electrodes were withdrawn from the targeted neuron, and the slices were 

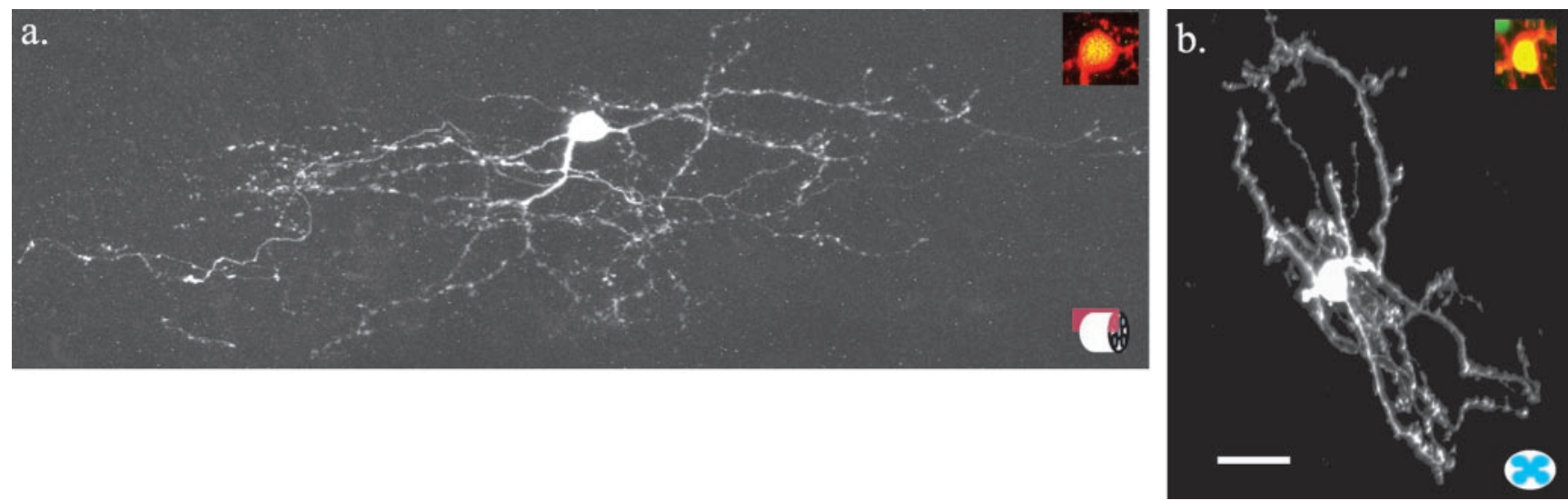

Figure 3. Morphology of biocytin-filled SG-GFP neurons expressing GFP. $a$, Maximum projection confocal image of a representativeneuron in a sagittal spinal section. $b$, Maximum projection confocal image of a representative neuron in a transverse section. Insets are merged images of GFP signal (green) and biocytin signal (red). Orange-yellow indicates biocytin labeling of a GFP-expressing neuron. SG-GFP neurons are morphologically equivalent to central neurons. Dorsal is shown at the top. Scale bar, $25 \mu \mathrm{m}$.

immersed in $4 \%$ paraformaldehyde. After 24-48 hr of fixation, the tissue was cryoprotected by immersion in a $30 \%$ sucrose phosphate buffer. To detect the biocytin, the tissue was washed and incubated with Texas Red streptavidin (1:800; Vector Laboratories).

Imaging. The fluorescent signals of the spinal cord sections were collected as $z$-series images using a confocal microscope [Leica (Nussloch, Germany) DM IRBE, DM RXE; Leica TCSNT]. Signal bleed-through was always checked and, when present, was eliminated by sequential capture of the fluorescent signals. Photoshop 6.0 (Adobe Systems, Mountain View, CA), Leica confocal software, Slicer 1.1 (Fortner Research, Sterling, VA), and Volocity 2.0 (Improvision, Lexington, MA) were used to analyze the confocal images. Laminar boundaries of the SG were determined from cytoarchitecture and, when possible, immunohistochemical markers. Positive immunoreactivity was defined as fluorescent signals with intensities at least $20 \%$ greater than background. Colocalization was judged on the basis of three-dimensional alignment of positive immunoreactivity of two fluorescent signals.

\section{Electrophysiology}

Electrophysiological experiments were performed as described in Grudt and Perl (2002) with minor alterations. Briefly, adult mice were killed by decapitation, and a laminectomy was performed. The tissue was placed in sucroseartificial CSF (ACSF) solution containing (in mM): 223 sucrose, $25 \mathrm{NaHCO}_{3}, 1.2 \mathrm{NaH}_{2} \mathrm{PO}_{4}$, $3.6 \mathrm{KCl}, 2 \mathrm{CaCl}_{2}, 1 \mathrm{MgCl}_{2}, 0.4$ ascorbic acid, 2 pyruvate, and $11 \mathrm{D}$-glucose. The vertebrae and dura were removed, and the dorsal roots were cut just central to the dorsal root ganglion. The lumbosacral region of the spinal cord was sectioned in ice-cold sucrose-ACSF using a vibrating microtome (Vibratome 3000) into transverse $(600-1000 \mu \mathrm{m})$ or sagittal $(200-500 \mu \mathrm{m})$ slices with attached dorsal rootlets. The slices were incubated for a minimum of $1 \mathrm{hr}$ in standard ACSF containing (in mM): $125 \mathrm{NaCl}, 26$ $\mathrm{NaHCO}_{3}, 1.25 \mathrm{NaH}_{2} \mathrm{PO}_{4}, 2.5 \mathrm{KCl}, 2 \mathrm{CaCl}_{2}, 1 \mathrm{MgCl}_{2}, 0.4$ ascorbic acid, 2 pyruvate, and $26 \mathrm{D}$-glucose, and equilibrated with $95 \% \mathrm{O}_{2}$ and $5 \% \mathrm{CO}_{2}$. The slices were transferred to a recording chamber (Warner Instruments, Hamden, CT), and a segmental dorsal root was drawn into a suction
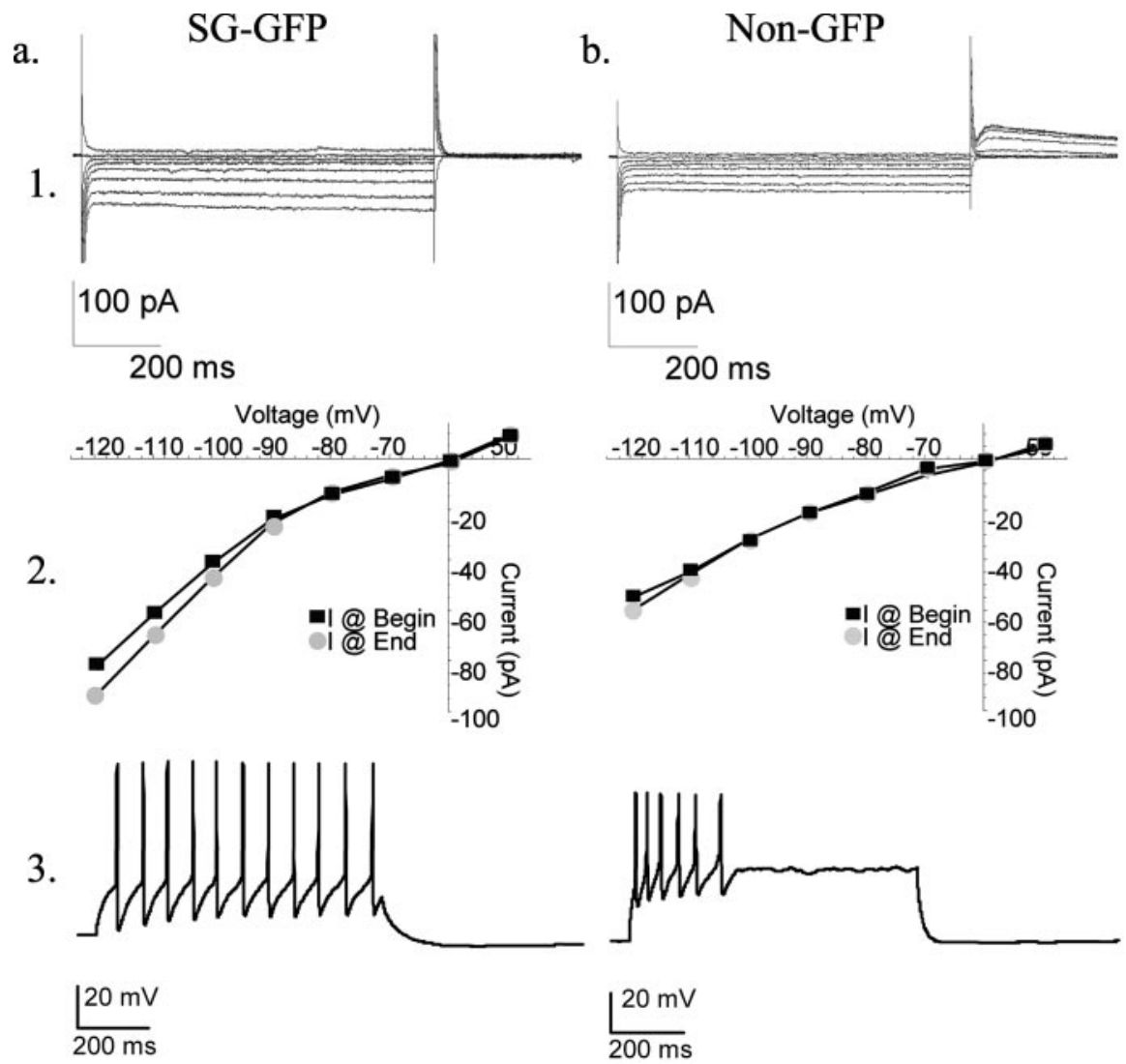

Figure 4. Electrophysiological properties of mouse neurons. Row 1, Current versus voltage traces. Row 2, Current versus voltage plots taken from the beginning and end of each voltage step. Row 3, Action potential generation by a prolonged (1 sec) depolarization. $a$, A representative SG-GFP neuron showing small inward rectification (changing slope of current-voltage plots), slight $I_{\mathrm{h}}$-like currents (hyperpolarization-activated depolarizing currents), and a tonic action potential generation pattern in response to a maintained depolarization. $b$, An example of an SG neuron in the Prp-GFP mouse lacking GFP. Note the absence of $I_{h}$, the presence of $I_{A}$ (outward tail current), and the transient pattern of action potential generation in response to a maintained depolarization. Neurons were held at $-60 \mathrm{mV}$ during voltage-clamp recordings.

stimulating electrode. Gravity-fed ACSF suprafused the tissue at $\sim 6 \mathrm{ml} /$ min: pharmacological compounds were added to ACSF. A fluorescent upright microscope (Leica DMLFS) and a charge-coupled device camera system (Cohu, San Diego, CA) were used to identify fluorescence during electrophysiological recording. Infrared imagery was used to direct a recording pipette, backfilled with $0.5 \%$ biocytin (Sigma), to SG neurons. SG-GFP neurons were targeted by directing the recording pipette to an 
infrared image of an SG neuron that had a corresponding fluorescent signal. The internal pipette solution contained (in $\mathrm{mM}$ ): 130 potassium gluconate, $5 \mathrm{NaCl}, 1 \mathrm{CaCl}_{2}, 1 \mathrm{MgCl}_{2}, 10 \mathrm{HEPES}$, and $4 \mathrm{Na}_{2} \mathrm{ATP}$. After acquiring gigaohm seals, tight-seal, whole-cell recordings were obtained. An Axopatch 1D (Axon Instruments, Foster City, CA) and pClamp6 software (Axon Instruments) were used to acquire data. Dorsal root volleys were initiated by pulses from a constant-voltage pulse generator applied through the suction electrode. Successive dorsal root-evoked responses with latencies showing a coefficient of variation $<2 \%$ were considered to be monosynaptic ( $\mathrm{Li}$ and Perl, 1994). Conduction velocity for the fastest monosynaptic dorsal root inputs was estimated from the response latency and the dorsal root length $(5 \mathrm{~mm})$. One millisecond was attributed to synaptic delay. The primary afferent input was defined on the basis of estimated conduction velocities: $\mathrm{A} \alpha \beta,>5 \mathrm{~m} / \mathrm{sec} ; \mathrm{A} \delta, 4-0.8$ $\mathrm{m} / \mathrm{sec} ; \mathrm{C},<0.8 \mathrm{~m} / \mathrm{sec}$ (Grudt and Perl, 2002). Current-voltage relationships were constructed from currents required to voltage clamp a neuron for $800 \mathrm{msec}$ at potentials ranging from -50 to $-120 \mathrm{mV}$ (at $10 \mathrm{mV}$ increments). In current-clamp mode, action potential generation patterns were established for near-threshold to suprathreshold depolarizing voltage steps $(1 \mathrm{sec})$ through the recording pipette. Data analysis was performed using Clampfit 8 (Axon Instruments). Colocalization of the fluorescent signals representing biocytin and GFP were used to establish that a recording came from an SG-GFP neuron. If biocytin labeling proved inadequate, the appearance of GFP fluorescence in the pipette electrode was used to establish that a whole-cell recording was from an SG-GFP neuron.

\section{$c$-Fos experiments}

Similar volumes of $5 \%$ formalin $(n=6)$ or $0.9 \% \mathrm{NaCl}(n=4)$ were injected subcutaneously into the dorsal surface of the right hindpaw of 4 to 6-week-old awake male mice. Behavior of the animals was monitored for $2 \mathrm{hr}$ after the injections. At $2 \mathrm{hr}$ postinjection, the animals were decapitated, and the spinal cords were removed, as described for the electrophysiological experiments, and immersed in $4 \%$ paraformaldehyde. Confocal microscopy was used to assess colocalization as described above. The number of SG-GFP neurons that expressed c-Fos protein was counted for the ispilateral and contralateral sides of 20 transverse spinal cord sections $(30 \mu \mathrm{m})$ from two animals for each condition: no injection, a saline injection, and a formalin injection. Chance differences in values were evaluated by statistical tests mentioned in Results $(p<0.05$ was taken as significant).

\section{Results}

\section{Distribution of GFP-expressing spinal cord cells}

On cytoarchitectural grounds, the majority of GFP-expressing cells were located in the outer part of Rexed's (1952) lamina II, the zone corresponding to the outer SG (Fig. $1 a, b$ ). It has been proposed that the binding of IB4 by primary afferent fibers delineates outer lamina II ( $\mathrm{II}_{\mathrm{o}}$ ) in the mouse (Woodbury et al., 2000). However, several reports suggest that IB4 binding marks inner lamina $\left.\mathrm{II}_{(\mathrm{II}}\right)$, not $\mathrm{II}_{\mathrm{o}}$ (Silverman and Kruger, 1988, 1990). Three-quarters (1511 of 2006) of SG-GFP somata identified in 120 transverse thoracic, lumbar, and sacral spinal sections were located in regions rich in IB4 binding (Fig. 1b). The majority of SG-GFP somata were located between the ventral boundary of substance P immunoreactivity and the ventral boundary of IB4 binding. However, the laminar overlap of various primary afferent markers and the complex neurite branching patterns of SG neurons make it difficult to draw valid conclusions about connections from the location of neuronal somata. The GFPexpressing cells were spaced at $\sim 50 \mu \mathrm{m}$ intervals across the mediolateral axis of the spinal cord (Fig. 1a). This GFP distribution pattern was present in all of the Prp-GFP animals examined and was relatively consistent over the length of the spinal cord.

A rare GFP cell was noted ventral to the SG in the deep dorsal horn (supplemental Fig. 1; available at http://www.med.unc.edu/ physiology/fac_hantman.htm). In addition, ependymal cells lin-

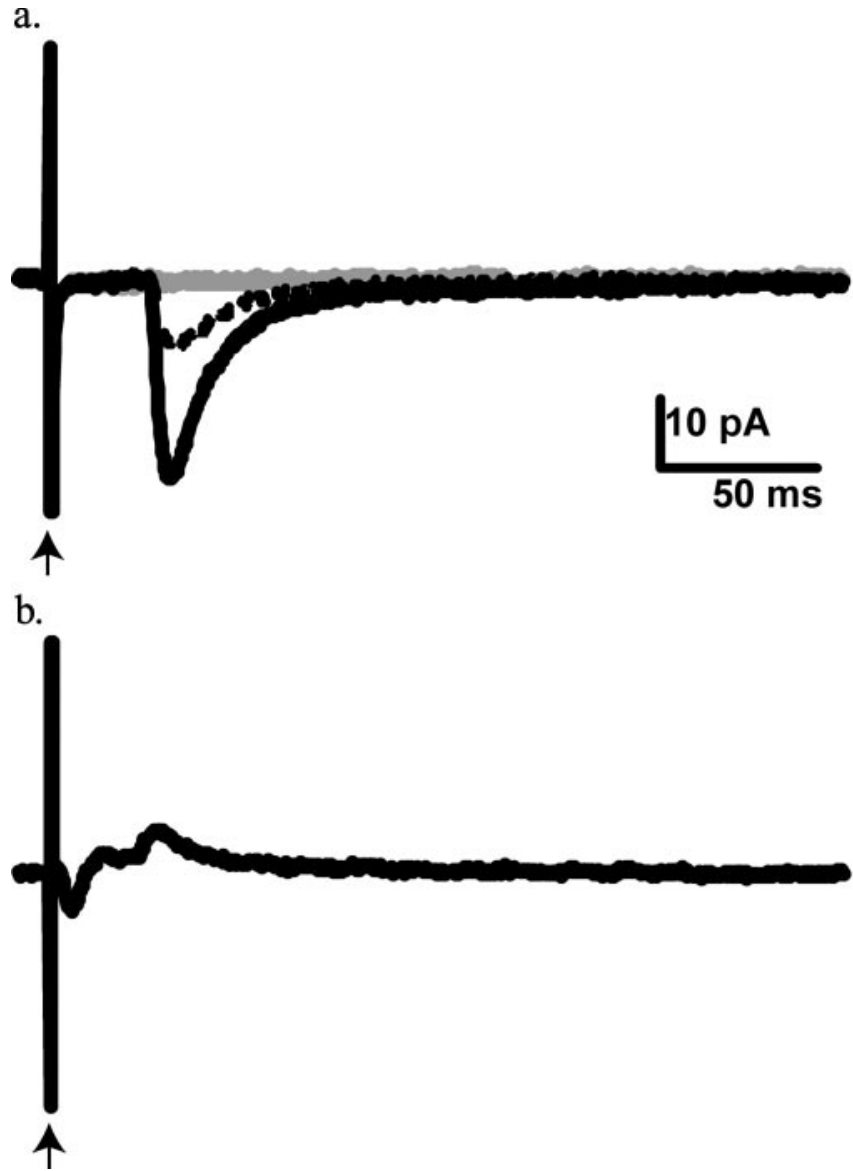

Figure 5. Responses of SG neurons in the Prp-GFP mouse to dorsal root stimulation. Each trace represents the average of 10 successive stimulations. Gray traces represent dorsal root responses during the application of $10 \mathrm{~mm}$ CNQX. Dashed traces represent dorsal root responses during wash period. $a$, The lack of variation in the long latency responses of SG-GFP neurons is consistent with a monosynaptic C-fiber input. The block of response by CNQX suggests mediation by AMPA-type glutamate channels. $b$, The dorsal root response of an SG neuron lacking GFP expression shows a briefer latency and a more complex form, indicating input from $A \delta$ fibers and a mixture of excitation and inhibition. Arrow indicates the stimulation artifact.

ing the central canal throughout the length of the spinal cord expressed GFP.

Neuronal identity of the GFP-expressing spinal cord cells The neuronal nature of the GFP-expressing cells was evaluated by examining for the NeuN protein (Mullen et al., 1992; Todd et al., 1998). All of the SG-GFP cells identified in $30 \mu \mathrm{m}$ transverse sections ( $n=180$ sections) from thoracic, lumbar, and sacral spinal cord regions of three animals (at least 150 SG-GFP neurons per region) were immunoreactive to an antibody to NeuN (Fig. 1c). The SG-GFP cells also universally expressed immunoreactivity to an antibody to neuron-specific enolase, another neuronal marker (Schmechel et al., 1978). Using NeuN-positive cells to estimate of the number of SG neurons, GFP-labeled neurons represented $6 \%$ of SG neurons in the thoracic region, $8 \%$ in the lumbar region, and $7 \%$ in the sacral region. Cells lining the central canal were the only GFP-expressing cells in the spinal cord that lacked positive NeuN immunoreactivity.

\section{Classification of mouse SG neurons}

The distribution of GFP-labeled neurons and the morphological similarity of the individual GFP cells (Fig. 1d) raised the possibility that the SG-GFP neurons represented a single subtype. To 
match the SG-GFP neurons to an existing SG neuronal classification scheme, we examined the mouse SG for analogy to that of other species. To this end, detailed morphological and electrophysiological features of individual SG neurons were determined by performing tight-seal, wholecell electrophysiological studies with biocytin-filled recording electrodes. Histochemical detection of intracellular biocytin was used to visualize neuronal morphology. Mouse SG neurons not labeled by GFP $(n=41)$ were morphologically and electrophysiologically diverse, representing examples (Fig. 2) of categories equivalent to those described for hamster SG neurons (Grudt and Perl, 2002). One GFP-negative type fits the vertical-cell category, exhibiting a high frequency of miniature postsynaptic currents, a delayed action potential to a depolarization step, and fan-like dorsoventrally oriented neurites (Fig. 2a). Another type had extensive processes oriented in the mediolateral axis, characteristic of medial-lateral cells (Fig. 2b). Radial-like cells with spoke-like neurites emanating from the soma in all of the directions were also noted (Fig. 2c). GFP-negative cells in the mid-SG with moderately long $(\sim 200$ $\mu \mathrm{m})$ neurites in the rostrocaudal direction appeared similar to hamster central cells (Fig. 2d). The latter included both transient and tonic varieties. These similarities of SG component neurons between mouse and other species encourage applicability of existing SG cytoarchitectural concepts to the mouse.

\section{Morphological profile of individual SG-GFP neurons}

The somata of SG-GFP neurons labeled by biocytin $(n=21)$ were oval in shape with the longest dimension $(13 \pm 3 \mu \mathrm{m})$ in the rostrocaudal axis (Fig. 3). Rostrocaudal dendrites averaged $194 \pm$ $91 \mu \mathrm{m}$ in sagittal sections (Fig. $3 a$ ). Dendrites extending in the medial and lateral direction were rare and, when present, were short $(44 \pm 24 \mu \mathrm{m}$ ) in transverse sections (Fig. $3 b$ ). The mean extent of dorsoventral processes was $92 \pm 43 \mu \mathrm{m}$ (Fig. 3). Biocytin-labeled axons of SG-GFP neurons did not appear to project outside the SG (a three-dimensional reconstruction of an SG-GFP neuron appears in supplemental Fig. 2, available at http:// www.med.unc.edu/physiology/fac_hantman.htm). On the basis of the morphological characteristics of the SG neuronal subtypes defined in the hamster and rat (Grudt and Perl, 2002; Lu and Perl, $2003)$, all of the SG-GFP neurons labeled by biocytin $(n=21)$ fit the morphological characteristics of the central-cell category (Ramon y Cajal, 1909).

\section{Electrophysiological characteristics of SG-GFP neurons}

The input resistance of SG-GFP neurons averaged $158 \pm 68 \mathrm{M} \Omega$ $(n=28)$ and the mean resting membrane potential was $-48 \pm 7$ $\mathrm{mV}(n=28)$. Twenty-five of 28 SG-GFP neurons exhibited inward rectification (Fig. 4a1,2). Most SG-GFP neurons (20 of 28) expressed a hyperpolarization-activated inward current $\left(I_{\mathrm{h}}\right)$; however, none $(n=28)$ showed a hyperpolarization-activated outward tail current $\left(I_{\mathrm{A}}\right)$. SG-GFP neurons initiated action potentials on depolarization without delay (27 of 28 ); all (28 of 28 ) fired tonically
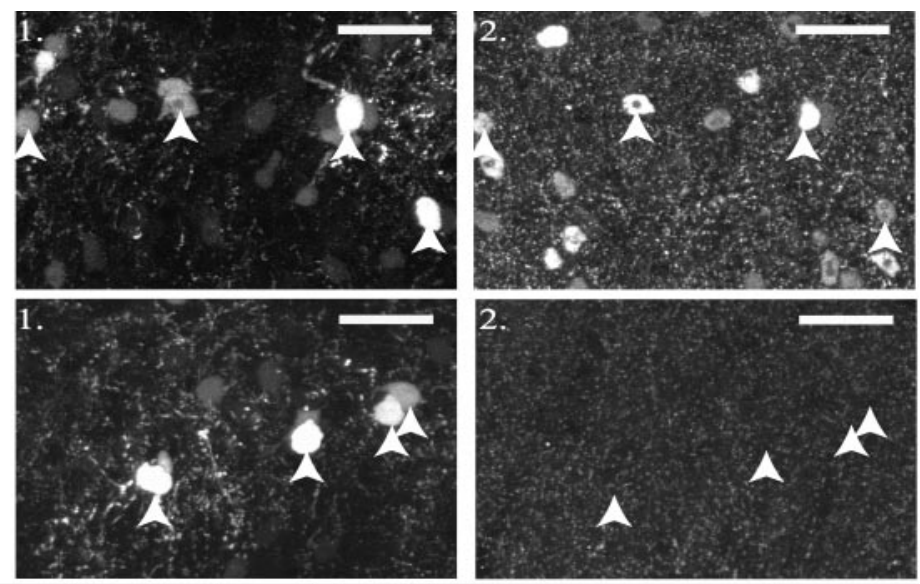
os positive SG-GFP newrons per section (20 transverse sections)

Saline $\quad$ Formalin

\begin{tabular}{|c|c|c|c|}
\hline \multicolumn{2}{|c|}{ Saline } & \multicolumn{2}{c}{ Formalin } \\
\hline 1 & 2 & 1 & 2
\end{tabular}

2

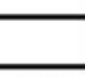
$\mu \mathrm{m}$. c, Mean numbers ( \pm SEM) of SG-GFP neurons per section expressing c-Fos for saline and formalin injections. Paired twotailed $t$ test $p$-values are shown for difference between the sides ipsilateral and contralateral to the formalin injections.

throughout a depolarizing pulse. Most SG-GFP cells (26 of 28) generated action potentials at relatively constant interspike intervals (Fig. 4a3).

In contrast to SG-GFP neurons, electrophysiological properties of nearby, randomly selected SG neurons without GFP labeling exhibited a much broader range of features (Yoshimura and Jessell, 1989; Grudt and Perl, 2002). The input resistances of a sample of GFP-negative SG neurons ranged widely from 114 to $817 \mathrm{M} \Omega(n=41)$; their resting membrane potentials varied from -40 to $-72 \mathrm{mV}$. Some GFP-negative SG neurons exhibited inward rectification (13 of 41), approximately one-half (23 of 41) showed no rectification, and 5 of 31 exhibited outward rectification. $I_{\mathrm{h}}$ currents were apparent in 25 of 41 GFP-negative SG neurons, whereas $I_{\mathrm{A}}$ currents were noted in 8 of 41 (Fig. $4 b 1,2$ ). A few GFP-negative SG neurons (6 of 41) discharged action potentials in bursts; a number (18 of 41) had irregular interspike intervals (Fig. 4b3).

\section{Dorsal root input to the SG-GFP neurons}

Dorsal root stimulation evoked responses in SG-GFP neurons (Fig. 5a). In 9 of 10 SG-GFP neurons, consecutive dorsal rootevoked responses varied little in latency (coefficient of variation, $<2 \%$ ), indicative of a monosynaptic connection. The conduction velocity of the dorsal root fibers underlying this presumed monosynaptic input $(n=9)$ ranged from 0.10 to $0.25 \mathrm{~m} / \mathrm{sec}$ (average, $0.15 \pm 0.06 \mathrm{~m} / \mathrm{sec}$ ), a value consistent with C-fiber input. The dorsal root-evoked responses were reversibly blocked by 6-cyano-7-nitroquinoxaline-2,3-dione (CNQX) (10 $\mu \mathrm{M})$, suggesting mediation by AMPA-type glutamate receptors (Fig. $5 a$ ). Dorsal root stimulation failed to generate monosynaptic or polysynaptic responses at latencies that would be indicative of a 

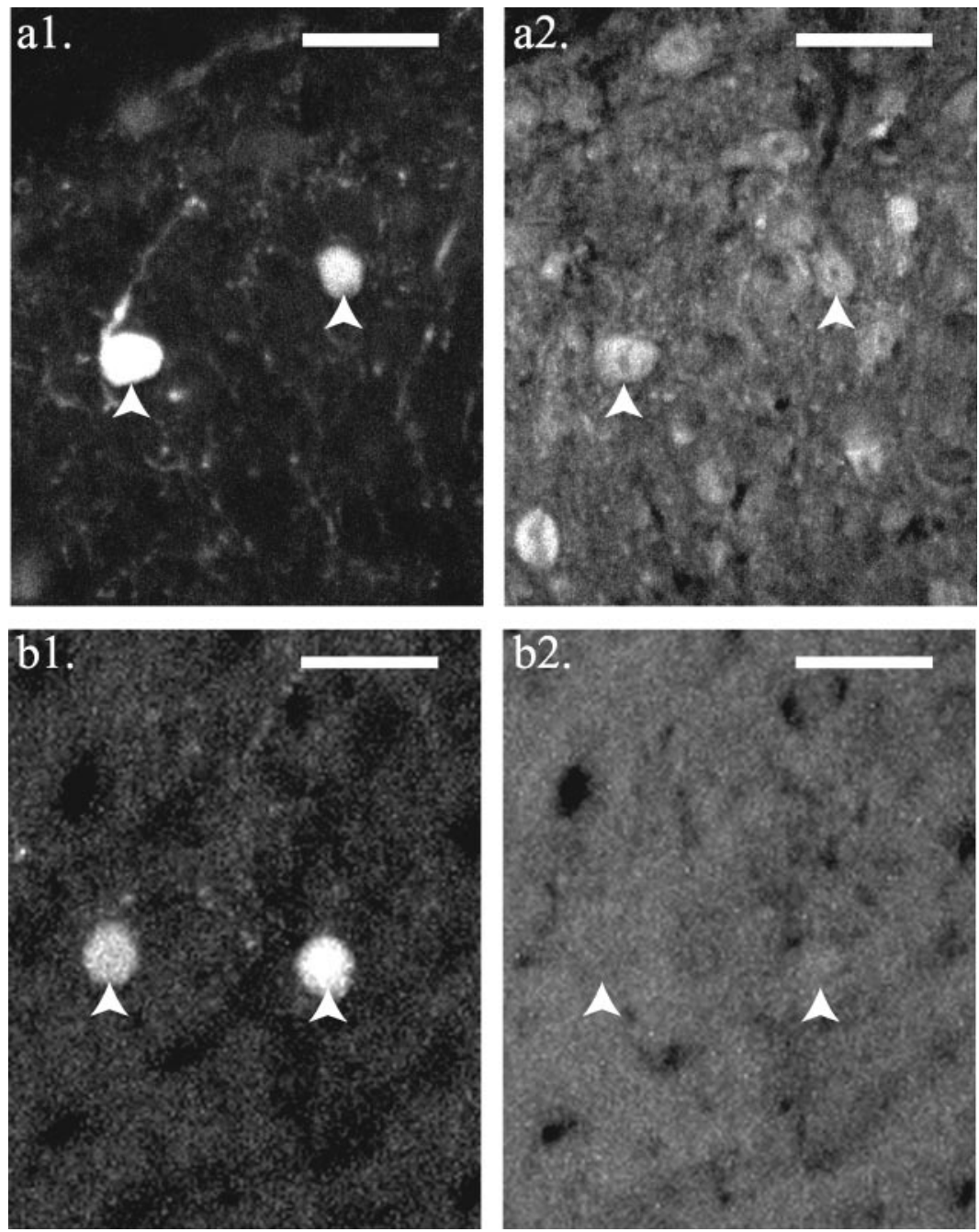

Figure 7. The majority of SG-GFP neurons express immunoreactivity to GABA antibodies. This immunoreactivity was blocked by preabsorption with a GABA-BSA conjugate. a1, b1, GFP signal from transverse sections of lumbar spinal cord. $a 2, b 2$, Immunoreactivity to a GABA antibody in sections shown in $a 1$ and $b 1$, respectively. The tissue section in $b 1$ and $b 2$ was preabsorbed with a GABA-BSA conjugate $(2.5 \mathrm{mg} / \mathrm{ml})$. Somata of GFP neurons are indicated by arrows. Dorsal is shown at the top. Scale bars, $25 \mu \mathrm{m}$.

myelinated fiber input to SG-GFP neurons. In contrast, responses to activation of dorsal root fibers conducting at $\mathrm{A} \delta$ velocities were noted in GFP-negative SG neurons adjacent to SGGFP cells (Fig. 5b).

\section{Noxious stimuli activate SG-GFP neurons}

Substantial noxious stimulation of peripheral tissue can cause the upregulation of c-Fos protein in a population of SG neurons (Hunt et al., 1987). To determine whether the GFP-positive neurons upregulate c-Fos protein in response to noxious stimuli, formalin was injected into the dorsal surface of the right hindpaw (Tjolsen et al., 1992) of Prp-GFP mice $(n=6)$. In control animals $(n=2)$, SG-GFP neurons did not exhibit c-Fos expression ( 0 of 633 , and 0 of 922). Figure 6 shows that a significantly higher number of c-Fos-expressing SG-GFP neurons appeared ipsilateral to a formalin injection than contralateral (two animals; $p<$ 0.00005; two-tailed paired $t$ test). Ipsilateral c-Fos expression in SG-GFP neurons in animals receiving a formalin injection $(n=$ $6)$ was strikingly greater than in animals $(n=4)$ receiving a saline injection $(p<0.05$; two-tailed, two-sample unequal variance $t$ test); see Figure $6 c$. Approximately $10 \%(n=2 ; 49$ of 506 , and 57 of 477) of SG neurons that exhibited c-Fos immunoreactivity after formalin injection also expressed GFP.

\section{SG-GFP neurons are GABAergic}

SG-GFP neurons exhibit immunoreactivity to three different antibodies established to stain neurons containing GABA as a synaptic agent. These antibodies labeled known GABAergic cells such as the Renshaw cells of the ventral horn. More than $80 \%$ (703 of 843) of SG-GFP neurons showed GABAlike immunoreactivity (Fig. 7a1,2). This proportion is probably an underestimate, because GABA antibodies have limited penetration into tissue. SG-GFP neurons labeled by GABA antibodies constituted $\sim 11 \%$ (294 of 2652) of a sample of SG neurons exhibiting GABA immunoreactivity. Preabsorption with a GABA-BSA conjugate completely blocked SG-GFP neuronal immunoreactivity to the GABA antibodies (Fig. 7b1,2) but did not affect immunoreactivity to a calcitonin gene-related peptide antibody in the same tissue.

\section{Discussion}

SG-GFP neurons have features of tonic central cells

Ramon y Cajal (1909) called a category of morphologically distinctive dorsal horn neurons central cells because of their usual location in the middle of the SG. Dendrites of central cells are restricted in the dorsoventral and mediolateral axes and are of moderate lengths $(\sim 200-300 \mu \mathrm{m})$ in the rostrocaudal plane (Gobel, 1975; Grudt and Perl, 2002). Central-cell axons appear confined to the dorsal horn. Differences in action potential generation patterns during a step depolarization separates the central-cell group into two classes (Grudt and Perl, 2002). Some central cells generate action potentials throughout a prolonged step depolarization (tonic type), whereas others only produce action potentials during the initial phase of the depolarization (transient type). Tonic central cells also exhibit inward rectification and $I_{\mathrm{h}}$ currents. Finally, a proportion of Golgi-stained neurons, morphologically resembling central cells, are reported to be GABAergic (Todd and McKenzie, 1989).

The morphological and electrophysiological features of the SG-GFP neurons suggest that they represent tonic central cells. Cytoarchitecture (size and density of neurons) and the relationship of the location of SG-GFP somata to IB4 binding and the ventral border of immunoreactivity to substance $\mathrm{P}$ locate these cells in the zone containing the central class of SG neurons. The cellular morphology of the SG-GFP neurons, including the local projection of their axons, also corresponds to features of central cells in other species. The small inward rectification, small $I_{\mathrm{h}}$ currents, and the tonic generation of action potentials by a suprathreshold depolarization fit features of tonic central cells. However, the apparent limitation of direct dorsal root input to C-fibers may indicate that the SG-GFP neurons are a subset of 
tonic central cells, because, in some other rodents, tonic central cells receive monosynaptic dorsal root input from $\mathrm{A} \delta$ fibers as well (Grudt and Perl, 2002). Furthermore, in the Prp-GFP transgenic mouse, some tonic SG central neurons do not express GFP.

\section{C-fiber input to inhibitory neurons}

Our observations provide clues to a circuit possibly containing the SG-GFP neurons. Neurons that upregulate c-Fos protein in response to a noxious stimuli are argued to participate in nociceptive pathways (Hunt et al., 1987). SG-GFP neurons significantly upregulate c-Fos protein in response to a peripheral tissue injection of formalin, but not to a saline injection. The dorsal root response data suggest that dorsal root $\mathrm{C}$-fibers are a primary peripheral input to the SG-GFP neurons. Given the c-Fos observation and the fact that many somatic afferent $\mathrm{C}$-fibers are nociceptive in character, it is possible that suprathreshold inputs from nociceptors could evoke repetitive action potentials in SG-GFP neurons. Repetitive discharge in SG-GFP neurons could result in a GABAergic inhibitory action on other neurons of the superficial dorsal horn.

The case is made here that SG-GFP neurons constitute a homogenous population of tonic central cells. Their spaced, repeated location and their coherent functional attributes suggest that they represent a neuronal type with a repeating SG function. Repetition of a functional unit along the spinal cord would be consistent with a modular framework in which interconnected neurons are arranged to deal with related somatosensory inputs. Related inputs could arise from similar afferent sensory fibers or from comparable receptive fields. In such an organization, nests (sets) of neurons connected in a special functional way would subserve particular integrative functions related to selective primary afferent projections. This kind of modular arrangement would be comparable in principle with the neuronal columns of the somatosensory cerebral cortex and its relationship to primary afferent input.

Finally, these studies emphasize the relative ease of establishing features of a particular class of interneurons marked by the expression of a fluorescent protein. There are apparent advantages to this approach in analyzing function of a complex neuropil.

\section{References}

Clark FM, Proudfit HK (1991) The projection of noradrenergic neurons in the A7 catecholamine cell group to the spinal cord in the rat demonstrated by anterograde tracing combined with immunocytochemistry. Brain Res 547:279-288.

Dahlström A, Fuxe K (1965) Evidence for the existence of monoamine neurones in the central nervous system. II. Experimentally induced changes in the intraneuronal amine levels of bulbospinal neuron system. Acta Physiol Scand 64:1-36.

Gobel S (1975) Golgi studies of the substantia gelatinosa neurons in the spinal trigeminal nucleus. J Comp Neurol 162:397-415.

Gobel S (1978) Golgi studies of the neurons in layer II of the dorsal horn of the medulla (trigeminal nucleus caudalis). J Comp Neurol 180:395-414.

Grudt TJ, Perl ER (2002) Correlations between neuronal morphology and electrophysiological features in the rodent superficial dorsal horn. J Physiol (Lond) 540:189-207.

Hantman AW, von den Pol A, Perl ER (2002) A distinctive set of substantia gelatinosa neurons selectively expressing green fluorescent protein. Soc Neurosci Abstr: 28:259.9.

Hunt SP, Pini A, Evan G (1987) Induction of c-fos-like protein in spinal cord neurons following sensory stimulation. Nature 328:632-634.

Kumazawa T, Perl ER (1978) Excitation of marginal and substantia gelatinosa neurons in the primate spinal cord: indications of their place in dorsal horn functional organization. J Comp Neurol 177:417-434.

Lee KJ, Jessell TM (1999) The specification of dorsal cell fates in the vertebrate central nervous system. Annu Rev Neurosci 22:261-294.
Li J, Perl ER (1994) Adenosine inhibition of synaptic transmission in the substantia gelatinosa. J Neurophysiol 72:1611-1621.

Light AR, Perl ER (1979a) Reexamination of the dorsal root projection to the spinal dorsal horn including observations on the differential termination of coarse and fine fibers. J Comp Neurol 186:117-131.

Light AR, Perl ER (1979b) Spinal termination of functionally identified primary afferent neurons with slowly conducting myelinated fibers. J Comp Neurol 186:133-150.

Lu Y, Perl ER (2003) A specific inhibitory pathway between substantia gelatinosa neurons receiving direct C-fiber input. J Neurosci 23:8752-8758.

Mullen RJ, Buck CR, Smith AM (1992) NeuN, a neuronal specific nuclear protein in vertebrates. Development 116:201-211.

Müller T, Brohmann H, Pierani A, Heppenstall PA, Lewin GR, Jessell TM, Birchmeier C (2002) The homeodomain factor Lbxl distinguishes two major programs of neuronal differentiation in the dorsal spinal cord. Neuron 34:551-562.

PerlER (1984) Advances in pain research and therapy (Kruger L, Liebeskind JC, eds), pp 23-51. New York: Raven.

Price DD, Hayashi H, Dubner R, Ruda MA (1979) Functional relationships between neurons of marginal and substantia gelatinosa layers of primate dorsal horn. J Neurophysiol 42:1590-1608.

Proudfit HK, Anderson EG (1975) Morphine analgesia: blockade by raphe magnus lesions. Brain Res 98:612-618.

Ramon y Cajal S (1909) Histologie du systèm neuveux de l'homme et des vertébrés, Vol. 1. Paris: Maloine.

Ren K, Ruda MA (1994) A comparative study of the calcium-binding proteins calbindin-D28K, calretinin, calmodulin, and parvalbumin in the rat spinal cord. Brain Res Brain Res Rev 19:163-179.

Rexed B (1952) The cytoarchitectonic organization of the spinal cord in the cat. J Comp Neurol 96:415-495.

Schmechel D, Marangos PJ, Zis AP, Brightman M, Goodwin FK (1978) Brain enolases as specific markers of neuronal and glial cells. Science 199:313-315.

Silverman JD, Kruger L (1988) Lectin and neuropeptide labeling of separate populations of dorsal root ganglion neurons and associated "nociceptor" thin axons in rat testis and cornea whole-mount preparations. Somatosens Res 5:259-267.

Silverman JD, Kruger L (1990) Selective neuronal glycoconjugate expression in sensory and autonomic ganglia: relation of lectin reactivity to peptide and enzyme markers. J Neurocytol 19:789-801.

Sugiura Y, Lee CL, Perl ER (1986) Central projections of identified, unmyelinated (C) afferent fibers innervating mammalian skin. Science 234:358-361

Tjolsen A, Berge OG, Hunskaar S, Rosland JH, Hole K (1992) The formalin test: an evaluation of the method. Pain 51:5-17.

Todd AJ, Lewis SG (1986) The morphology of Golgi-stained neurons in lamina II of the rat spinal cord. J Anat 149:113-119.

Todd AJ, McKenzie J (1989) GABA-immunoreactive neurons in the dorsal horn of the rat spinal cord. Neuroscience 31:799-806.

Todd AJ, Spike RC (1993) The localization of classical transmitters and neuropeptides within neurons in laminae I-III of the mammalian spinal dorsal horn. Prog Neurobiol 41:609-645.

Todd AJ, Spike RC, Polgar E (1998) A quantitative study of neurons which express neurokinin-1 or somatostatin $s_{2} t_{2 \mathrm{a}}$ receptor in rat spinal dorsal horn. Neuroscience 85:459-573.

Tuckett RP, Wei JY (1987a) Response to an itch producing substance in cat. I. Cutaneous receptor populations with myelinated axons. Brain Res 413:87-94.

Tuckett RP, Wei JY (1987b) Response to an itch producing substance in cat. II. Cutaneous receptor populations with unmyelinated axons. Brain Res 413:95-103.

Vallbo AB, Olausson H, Wessberg J (1999) Unmyelinated afferents constitute a second system coding tactile stimuli of the human hairy skin. J Neurophysiol 81:2753-2763.

van den Pol AN, Ghosh PK, Liu RJ, Li Y, Aghajanian GK, Gao XB (2002) Hypocretin (orexin) enhances neuron activity and cell synchrony in developing mouse GFP-expressing locus coeruleus. J Physiol (Lond) 541:169-185.

Woodbury CJ, Ritter AM, Koerber HR (2000) On the problem of lamination in the superficial dorsal horn of mammals: a reappraisal of the substantia gelatinosa in postnatal life. J Comp Neurol 417:88-102.

Yoshimura J, Jessell TM (1989) Membrane potential of rat substantia gelatinosa neurons in vitro. J Neurophysiol 62:109-118. 